\title{
Spatially and temporally polarization shaped laser pulses for two-photon excited fluorescence
}

\author{
M. B. Hild, R. Gottschalk, K. Heyne, and A. Lindinger ${ }^{a}$ \\ Institut für Experimentalphysik, Freie Universität Berlin, Arnimallee 14, D-14195 Berlin, Germany
}

Received: date / Revised version: date

\begin{abstract}
We present simultaneous spatial and temporal polarization pulse shaping for two-photon excited fluorescence of dyes. A temporal pulse shaper and a subsequent spatial pulse shaper are used to generate laterally and axially tailored two-photon excited fluorescence profiles. The induced fluorescence is recorded for different polarization directions by utilizing the anisotropy of the dyes embedded in glycerol. We achieve an increased fluorescence contrast between different dyes by employing specific polarization shaped laser pulses and by taking polarization dependent emission into account. This versatile pulse shaping method is prospective for novel biophotonic imaging.
\end{abstract}

PACS. PACS-key discribing text of that key - PACS-key discribing text of that key

\section{Introduction}

In recent years laser pulse shaping received increasing attention due to its capability to control molecular processes and various applications emerged [1-8]. An important field of activity for tailored pulses is multiphoton excited fluorescence, where intrapulse interference is used to selectively excite different species [9], which is employed in multiphoton excited three-dimensional imaging of tissue [10]. In order to increase the shaping capabilities, additionally the modulation of the polarization was included into laser pulse shaping $[11,12]$. Another pulse shaping direction is the modification of the spatial laser profile by utilizing focussing lenses and a two dimensional liquid crystal array assembled to a spatial light shaper [13]. Spatial phase modulation of the liquid crystals yields well defined tailored beam profiles in the focal plane. Such beam profiles were employed for microstructuring [14], beam focussing in tissue [15], and high resolved spatial imaging [16]. For performing topical deep tissue imaging it is relevant to conduct axially resolved two-photon excited fluorescence measurements by modifying the focal depth employing specific spatial phase pattern.

As a next step, combined temporal and spatial laser pulse shaping was developed since it allows for modifying photo-induced processes, both temporally and spatially. Such tailored pulses were explored in initial experiments [17-19], yet the shaping properties were limited in these studies and particularly no polarization shaping was included. Here, we perform simultaneous temporal and spatial polarization pulse shaping for two-photon excitated fluorescence of molecules in a highly viscous liquid envi-

\footnotetext{
a e-mail: lindin@physik.fu-berlin.de
}

ronment. The experiments are conducted by utilizing a recently designed pulse shaper including a temporal and a subsequent spatial modulator. This enables independent temporal phase shaping of different spatial and polarization components. A polarization dependent fluorescence detection takes the anisotropy properties of the investigated molecules into account. The advanced pulse shaping method will be of note for novel biophotonic applications.

\section{Experimental}

The experimental setup is schematically shown in Fig. 1. The laser system includes a broadband titanium sapphire laser oscillator (Femtosource Compact, Femtolasers) with an average power of $350 \mathrm{~mW}$ and a repetition rate of $75 \mathrm{MHz}$ pumped by a frequency-doubled $\mathrm{Nd}: \mathrm{YVO}_{4}$ laser (Verdi V, Coherent, Inc.). The laser spectrum has a spectral full width at half maximum of about $80 \mathrm{~nm}$ with a center wavelength at $805 \mathrm{~nm}$. The laser beam is guided through a $4 \mathrm{f}$-pulse shaper having a computer controlled liquid crystal light modulator (SLM 640, Cambridge Research Instruments), which permits modulation of the light phase and polarization. In particular, the spectral phases can be independently modified on the two polarization axes at $\pm 45^{\circ}$ to the horizontal. The outgoing temporally shaped laser pulses pass a $\lambda / 2$-waveplate for rotating the polarization components to the vertical and horizontal direction. Subsequently, the beam is directed on a spatial 2D light modulator (PLUTO-NIR015-C, HOLOEYE Photonics AG), where the polarization components are projected on the vertical and horizontal axes of the 2D array in order to generate differing spatial shapes of the components. The spatial modulator has a $2 \mathrm{D}$ 


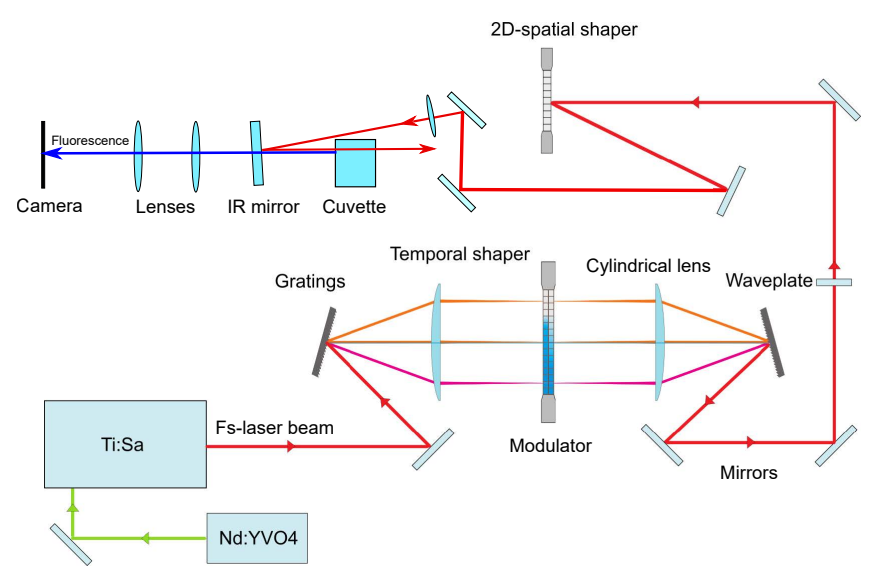

Fig. 1. Experimental scheme including a Ti:Sa laser, a temporal laser pulse shaper with gratings and a liquid crystal light modulator, a half-wave plate to rotate the light polarization, a 2D liquid crystal array for spatial beam modification, a cuvette, and a fluorescence detection setup. The fs-laser beam initially passes the temporal pulse shaper, subsequently its polarization is adjusted to correctly encounter the spatial pulse modulator, and then the beam is directed into a cuvette by using a low bandwidth dielectric mirror. The two-photon excited fluorescence passes through the mirror, and is collected and focussed on a camera.

array (15.36 mm x $8.64 \mathrm{~mm})$ with 1920 x 1080 liquid crystal elements and an attached mirror for reflection of the beam back through the liquid crystals. Subsequently, the laser beam is focussed in a 2f-setup by a lens ( $\mathrm{f}=300 \mathrm{~mm}$ ) located $300 \mathrm{~mm}$ behind the $2 \mathrm{D}$ modulator in order to realize spatially tailored profiles in the focal plane. Before the experiments, an optimization algorithm by phase resolved interferometric spectral modulation (PRISM) [22] is conducted in order to receive the phase retardances for the transform-limited pulses and thus a precise phase control. For the fluorescence experiments, the beam is focussed into a quartz glass cuvette filled with the dyes rhodamine B $(r h o B)$ or coumarin 47 (cou 47$)$ dispersed in glycerol. The two-photon excited fluorescence is collected and focussed on the detection camera (AW335, Ausdom Inc.) and a glass filter (BG 39) is used to reduce the stray light of the laser. Furthermore, a polarizer is placed in the fluorescence light path in order to be able to perform polarization dependent measurements.

\section{Results}

The first experiments are performed for top view detection of the two-photon excited fluorescence, whereby the detector faces the surface of the cuvette. To this end, a dielectric reflecting mirror for wavelengths around $800 \mathrm{~nm}$ is inserted to direct the beam on the surface of the cuvette with the fluorescence light transmitting the mirror (see Fig. 1). This top view geometry allows for simultaneous excitation and detection on the surface normal.
Two fluorescence spots are generated by utilizing the spatial pulse shaper. For this purpose, the horizontal polarization component is vertically upshifted by applying a linear spatial phase using the Zernike polynominal $Z_{1}^{-1}$ with a prefactor of 50 inscribed on the $2 \mathrm{D}$ array of the spatial pulse shaper, whereas the vertical polarization component is not shifted. Moreover, phase functions $\phi(\omega)=$ $\frac{b_{3}}{6}\left(\omega-\omega_{0}\right)^{3}$ with a third order phase factor $b_{3}=1 \cdot 10^{6}$ $\mathrm{fs}^{3}$ and differently tuned center frequencies $\omega_{0}$ are separately written on the two liquid crystal arrays of the temporal pulse shaper. These two perpendicularly polarized pulse components are temporally separated by 1.4 ps in order to reduce their overlap and hence prevent a possible disturbance of their polarization states. This leads to temporally differently tailored spots, where the upper spots show the two-photon fluorescence for the third order phase shaped horizontal polarization component at one center wavelength $\lambda_{0}=2 \pi c / \omega_{0}$ and the lower spots display the third order phase shaped vertical polarization component at another center wavelength. Hence, the spatially separated laser pulse components have different fluorescence intensities due to their particular phase functions [9]. These phases permit a major excitation of a specific dye by one spatial polarization component and simultaneously an excitation of another dye by the other spatial polarization component.

Figs. 2 and 3 display the results of polarization dependent two-photon excited fluorescence intensity measurements for rhodamine B and coumarin 47, respectively. Figs. 2(a) and 3(a) show camera images of two spots in a cuvette induced by spatially separated phase shaped polarization components. The upper spots present the twophoton fluorescence for the third order phase shaped horizontal polarization component at $\lambda_{0}=840 \mathrm{~nm}$ and the lower spots display the third order phase shaped vertical polarization component at $\lambda_{0}=800 \mathrm{~nm}$, respectively. Specificly, the polarization component for the upper spots maximizes the two-photon fluorescence of rhodamine B and the component for the lower spots the fluorescence of coumarin 47.

By inserting the polarizer in the fluorescence light path a strong reduction of one of the spots is visible in each image (see Figs. 2(b),(c) and 3(b),(c)). This can be explained by the anisotropy properties of the dyes embedded in highly viscous glycerol, which leads for these molecules to a mainly equal polarization of the excitation and fluorescence light. In particular, the fluorescence intensity is considerably reduced if the polarizer direction is perpendicular to the exciting polarization component. Hence, besides the above presented phase dependence the anisotropy has an additional influence on the fluorescence intensities. The combination of these features can be utilized to receive an increased contrast between the two dyes.

Fig. 4 presents the contrasts $c=\left(I_{r h B}-I_{\text {cou } 47}\right) /\left(I_{r h B}+\right.$ $\left.I_{\text {cou } 47}\right)$ for the two fluorescence spots. The insets below the graph depict the corresponding schematic 3D representations of the differently polarization tailored laser light fields at the two spatially separated spot positions. Yet, these light fields are coherently connected within the 

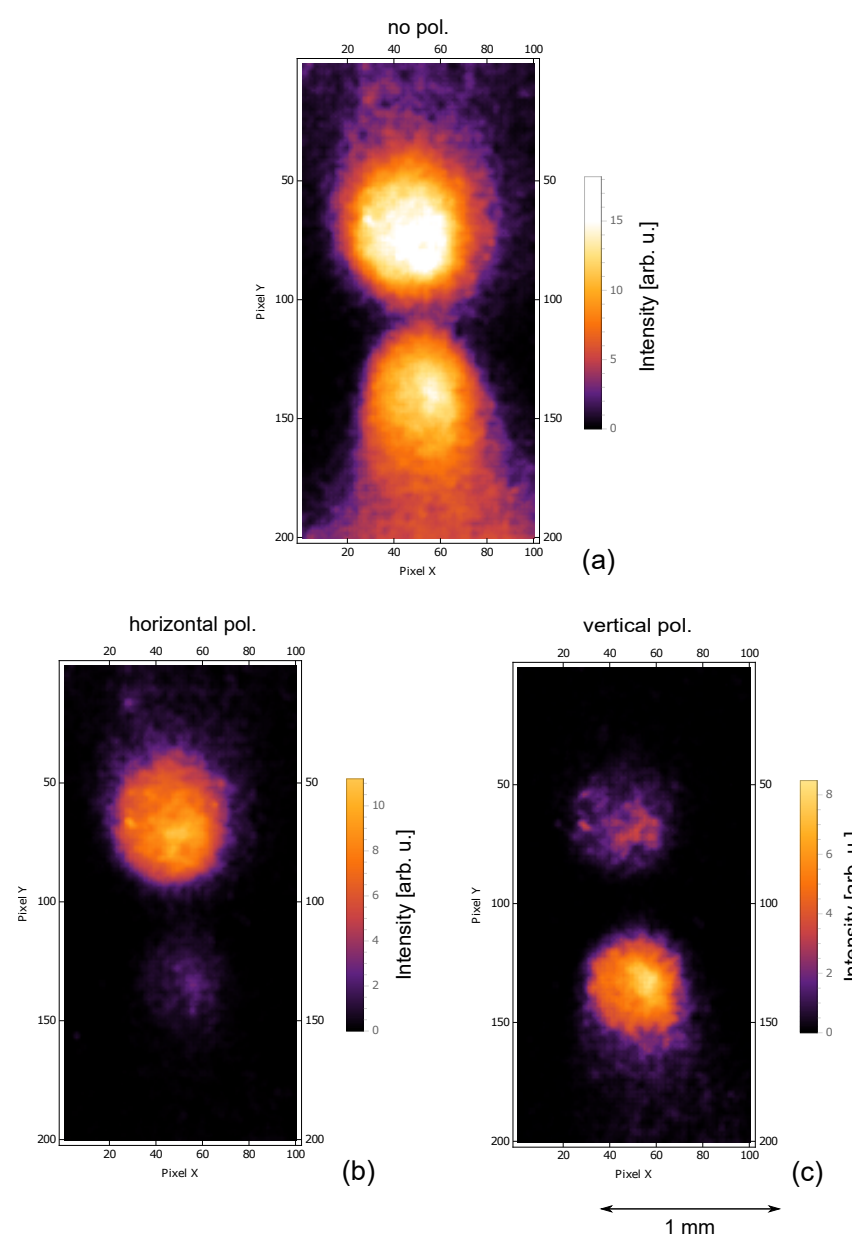

Fig. 2. Camera images of rhodamine B fluorescence in a cuvette excited by polarization tailored laser pulses, recorded with top view detection. (a) Image of two spots induced by spatially separated phase shaped polarization components, where the upper spot shows the fluorescence for the third order phase shaped horizontal polarization component at $\lambda_{0}=840 \mathrm{~nm}$ and the lower spot presents the third order phase shaped vertical polarization component at $\lambda_{0}=800 \mathrm{~nm}$. (b) Horizontal fluorescence polarization component of the measurement by introducing a polarizer. (c) Vertical fluorescence polarization component. The images show considerably differing spot intensities.

same laser pulse. For the case without polarizer one observes a higher contrast for the upper spot (position: 1 $\mathrm{mm}$ ) compared to the lower one (position: $0 \mathrm{~mm}$ ), leading to a contrast difference of about 0.2. Differing contrasts are obtained by including the polarizer with perpendicular orientations for the different dyes. For one combination the contrast change their order with the higher contrast for the lower spot, whereas for the other combination the contrast difference was further increased to a value of about 1.6. This is the case when the vertically polarized fluorescence data are subtracted from the horizontally polarized data (this is abbreviated by hor/vert in the legend of fig. 4 and vice versa by vert/hor). In the present experiment, the anisotropy effect on the contrast is larger than the effect of the differing phase functions. Thus, the contrast
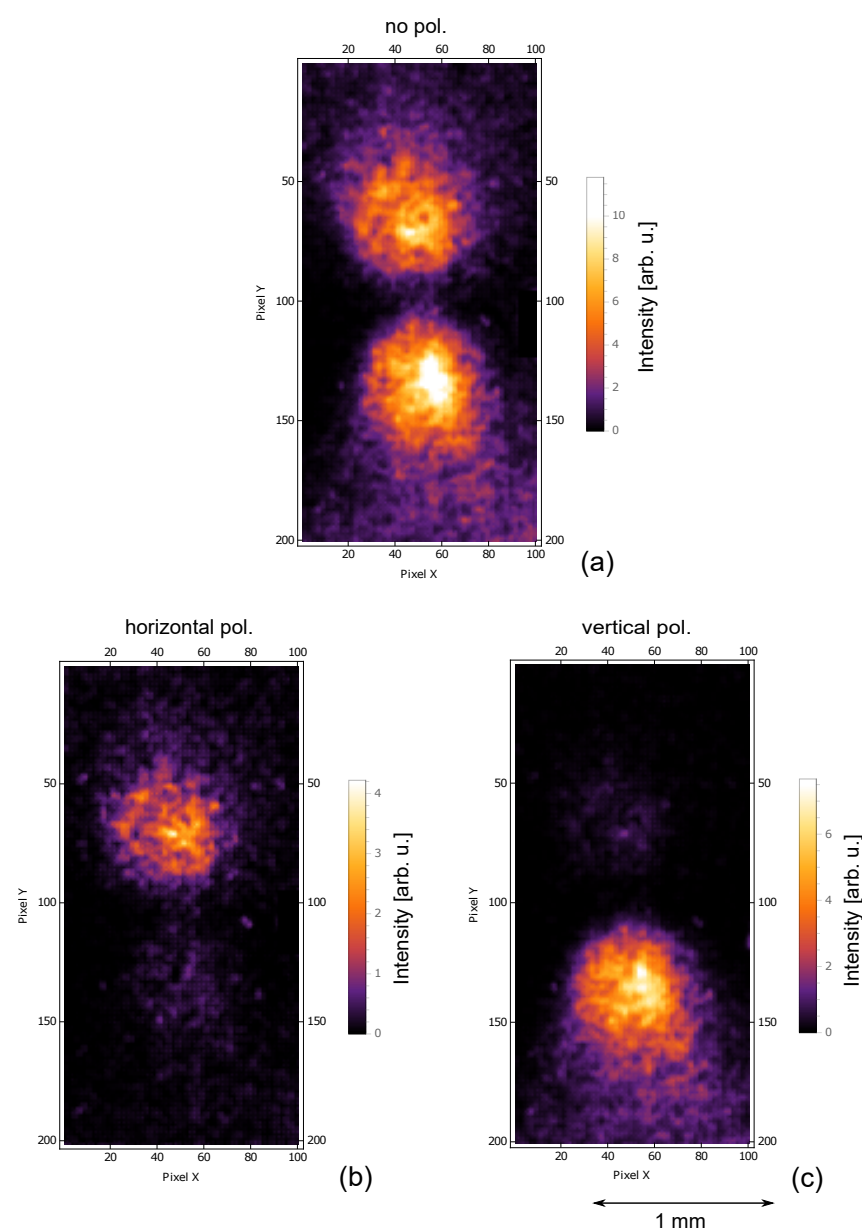

Fig. 3. Correspoding camera images of coumarin 47 fluorescence in a cuvette excited by polarization tailored laser pulses, recorded with top view detection. (a) Image of two spots, where the upper displays the fluorescence for the third order phase shaped horizontal polarization component at $\lambda_{0}=840 \mathrm{~nm}$ and the lower shows the third order phase shaped vertical polarization component at $\lambda_{0}=800 \mathrm{~nm}$. (b) Horizontal fluorescence polarization component by incluing a polarizer. (c) Vertical fluorescence polarization component.

difference can considerably be enhanced by favorably combining phase and anisotropy effects. This will be relevant for novel imaging applications.

It is also feasible to generate more complex spatial beam profiles by utilizing the spatial pulse shaper. Fig. 5(a) presents three fluorescence spots of the cuvette with rhodamine $\mathrm{B}$, where the two outer spots are produced by applying a sinusoidal phase grating $\phi(p)=A \sin (2 \pi p / \lambda)$ on the $2 \mathrm{D}$ modulator, with the amplitude $A=1.6$, the liquid crystal element number $p$, and the period $\lambda=45$ pixel. The central fluorescence spot is due to the not modulated vertically polarized component. The outer spots are weaker because the light of the horizontally polarized component is partitioned into both spots. It has to be mentioned that the two-photon fluorescence intensities depend on the square of the light intensities and therefore, predominantely the high intense light components will be visible in the image. Figs. 5(b) and 5(c) display the im- 


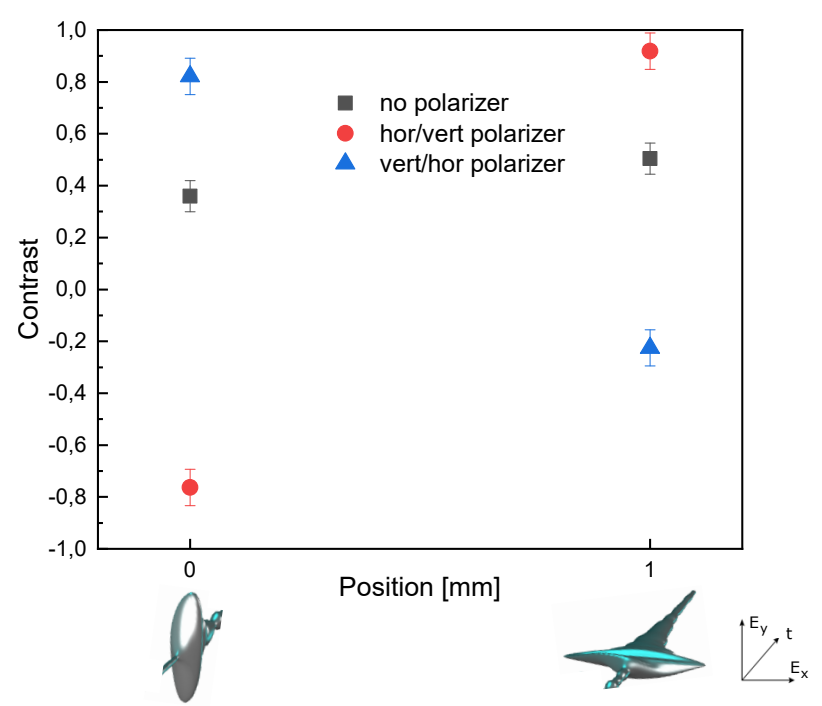

Fig. 4. Recorded contrast values between rhodamine $B$ and coumarin 47 for the two fluorescence spots at $\lambda_{0}=800 \mathrm{~nm}$ (position: $0 \mathrm{~mm}$ ) and $\lambda_{0}=840 \mathrm{~nm}$ (position: $1 \mathrm{~mm}$ ), respectively. Contrast values without polarizer and with perpendicular polarizer orientations for the differing dyes are presented. For a certain combination the contrasts change their order with the higher contrast for the lower spot (position: $0 \mathrm{~mm}$ ), whereas for the other combination the contrast difference is further enhanced. The insets at the bottom show schematic 3D representations of the differently polarization shaped light fields at the two corresponding spot positions within the same laser pulse.

ages for horizontal and vertical direction of the polarizer, respectively. Considerably reduced fluorescence intensities are obtained for the corresponding spots. By applying predefined $2 \mathrm{D}$ phase functions on the spatial modulator desired two-photon excitation pattern can be realized for applications.

\section{Conclusion}

An increased two-photon excited fluorescence contrast was received between different dyes by applying spatially and temporally polarization shaped laser pulses. To this end, a polarizer was used for recording the fluorescence polarization components, thereby utilizing the anisotropy of the dye molecules. The spatial and temporal polarization shaping method could particularly be employed to achieve an increased contrast of autofluorescing molecules. This novel approach facilitates polarization selective measurements at different spatial positions with temporally tailored light fields, which will give rise to new biophotonic applications.

\section{Acknowledgements}

The Klaus Tschira Foundation (KTS) is acknowledged for financial support (project 00.314.2017).
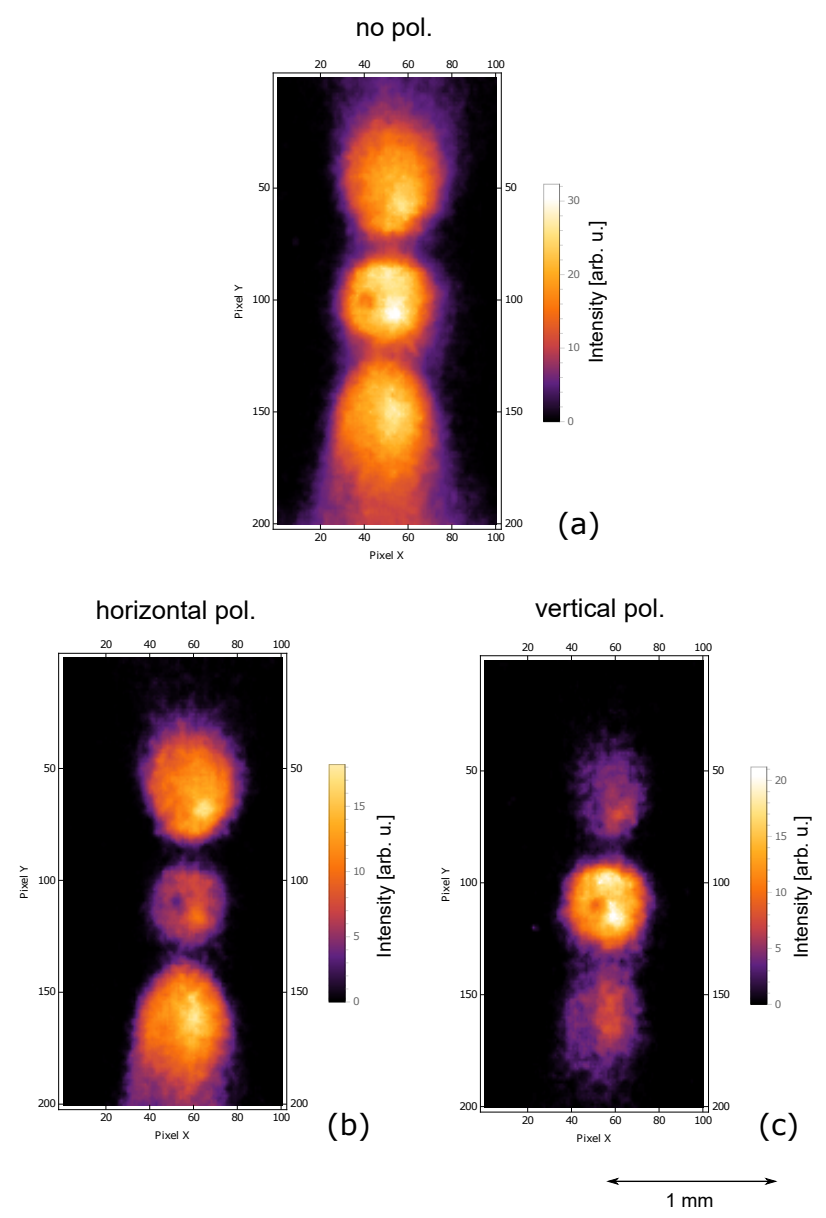

Fig. 5. (a) Two-photon excited fluorescence spots of the cuvette with rhodamine $B$, where the two outer spots are produced by applying a sinusoidal phase grating on the $2 \mathrm{D}$ modulator. The central fluorescence spot is due to the not modulated vertically polarized component. (b) Fluorescence image with horizontally oriented polarizer. (c) Image with vertically oriented polarizer.

\section{References}

1. R.S. Judson, H. Rabitz, Phys. Rev. Lett. 68, 1500 (1992)

2. P. Nuernberger, G. Vogt, T. Brixner, G. Gerber, Phys. Chem. Chem. Phys. 9, 2470 (2007)

3. M. Dantus, V.V. Lozovoy, Chem. Rev. 104, 1813 (2004)

4. A. Assion, T. Baumert, M. Bergt, T. Brixner, B. Kiefer, V. Seyfried, M. Strehle, G. Gerber, Science 282, 919 (1998)

5. T. Brixner, G. Gerber, ChemPhysChem 4, 418 (2003)

6. M. Aeschlimann, M. Bauer, D. Bayer, T. Brixner, F.J. Garcia de Abajo, W. Pfeiffer, M. Rohmer, C. Spindler, F. Steeb, Nature 446, 301 (2007)

7. A. Lindinger, C. Lupulescu, M. Plewicki, F. Vetter, A. Merli, S.M. Weber, L. Wöste, Phys. Rev. Lett. 93, 033001 (2004)

8. W. Wohlleben, T. Buckup, J.L. Herek, M. Motzkus, ChemPhysChem 6, 850 (2005)

9. V.V. Lozovoy, I. Pastirk, K.A. Walowicz, M. Dantus, J. Chem. Phys. 118, 3187 (2002)

10. W. Denk, J.H. Strickler, W.W. Webb, Science 248, 73 (1990) 
11. L. Polachek, D. Oron, Y. Silberberg, Opt. Lett. 31, 631 (2006)

12. F. Weise, A. Lindinger, Appl. Phys. B 101, 79 (2010)

13. C. Maurer, A. Jesacher, S. Bernet, M. Ritsch-Marte, Laser Photonics Rev. 5, 81 (2011)

14. N. Sanner, N. Huot, E. Audouard, C. Larat, J.P. Huignard, Opt. Lasers Eng. 45, 737 (2007)

15. A. Tanabe, T. Hibi, S. Ipponjima, K. Matsumoto, M. Yokoyama, M. Kurihara, N. Hashimoto, T. Nemoto, J. Biomed. Opt. 20, 101204 (2015)

16. G. Moneron, S. Hell , Opt. Exp. 17, 14567 (2009)

17. D.J. McCabe, A. Tajalli, D.R. Austin, P. Bondareff, I.A. Walmsley, S. Gigan, B. Chatel, Nature Communications 2, 447 (2011)

18. T. Feurer, J.C. Vaughan, R.M. Koehl, K.A. Nelson, Opt. Lett. 27, 652 (2002)

19. T. Feurer, J.C. Vaughan, K.A. Nelson, Science 299, 374 (2003)

20. M.J. Snare, F. Treloar, K.P. Ghiggino, P.J. Thistllethwaite, J. Photochem. 18, 335 (1982)

21. R.F. Kubin, A.N. Fletcher, J. Luminescence 27, 455 (1982)

22. T. Wu, J. Tang, B. Hajj, M. Cui, Opt. Express 19, 12961 (2011) 